\title{
Diversidad y ecología de mamíferos no voladores asociados a un sistema agro-productivo de cacao, Granja Yariguíes, Santander, Colombia
}

\author{
Diversity and ecology of non-volant mammals associated to a cacao agro-productive system, \\ Granja Yariguíes, Santander, Colombia
}

\author{
Carlos López-Ramírez ${ }^{1}$, Tatiana Restrepo-Quiroz ${ }^{2}$, Sergio Solari $^{1 *}$
}

\begin{abstract}
Resumen
Los sistemas agro-productivos se consideran una alternativa para amortiguar la pérdida de biodiversidad. En estos sistemas, algunos mamíferos son catalogados como plagas debido a las afectaciones que ejercen sobre estos cultivos, lo que se traduce en medidas de control que alteran las funciones que cumplen en dichos ecosistemas transformados. Durante un estudio realizado en la granja experimental Yariguíes, Santander, que incluye parcelas de producción ( $80 \%$ de la superficie) y zonas de reserva de bosque húmedo tropical ( $20 \%$ ), se determinó la diversidad de mamíferos no voladores, los posibles daños que ejercen, y los roles ecológicos que desempeñan. Se realizaron recorridos a través de las zonas cultivadas y de bosque, disponiendo cámaras automáticas y trampas de captura viva, además de buscar rastros como huellas, madrigueras o excretas. Adicionalmente, se evidenciaron y cuantificaron los daños en los cultivos que ejercen algunas especies de roedores. Se hallaron un total de 17 especies en cinco órdenes, representando 13 gremios; éstos evidencian los roles ecológicos que cumplen, y cómo pueden beneficiar los cultivos. La ardilla roja (Notosciurus granatensis) fue la especie que más atacó los frutos, con mayor predilección por las mazorcas maduras. Los daños por roedores en los cultivos presentaron baja incidencia ( $<5 \%$ en promedio), posiblemente porque las especies predadoras de cacao están siendo controladas por carnívoros que aún persisten en los bosques de reserva. Mientras que las zonas de reserva proveerían refugio y alimento para ciertas especies, las áreas de cultivo funcionarían como corredores, permitiendo un equilibrio clave para estos sistemas agro-productivos.
\end{abstract}

Palabras claves: ardilla roja, biodiversidad, fauna silvestre, roles ecológicos, Theobroma cacao L.

\begin{abstract}
Agro-productive systems are considered a valid alternative to diminish biodiversity loss. In these systems, some mammals are classified as pests due to their negative effect on crops, which results in control measurements that alter the functions that these species carry out on these transformed ecosystems. During a study at the Yariguies, Santander, experimental farm, which includes production plots $(80 \%$ of the surface area) and tropical rainforest reserve areas $(20 \%)$, we assessed the diversity of non-flying mammals, the possible damage they cause, and the ecological roles they play. We coursed through the cultivated and forest areas, setting automatic cameras and live traps, while searching for signs as tracks, burrows or feces. In addition, we detected and quantified the damage caused to the cacao crops by some rodent species; 17 species from five orders representing 13 guilds, were detected; these guilds show the ecological roles fulfilled
\end{abstract}

\footnotetext{
1. Instituto de Biología, Universidad de Antioquia, Medellín, Colombia

2. Compañía Nacional de Chocolates, Rionegro, Antioquia.

* Autor de correspondencia: <sergio.solari@udea.edu.co>
} 
by the mammals and how these roles can benefit the crops. The red-tailed squirrel (Notosciurus granatensis) was the species that attacked the cacao pods the most, showing higher preference for ripe ones. Damage by rodents at plots had a low frequency ( $<5 \%$ on average), possibly because predatory species of the pods are being controlled by the carnivores still present in the forest reserve. While forest reserve areas could provide refuge and food resources to some species, productive plots could act as corridors, allowing a key equilibrium for these agro-productive systems.

Keywords: red-tailed squirrel, biodiversity, wild fauna, ecological roles, Theobroma cacao L.

\section{INTRODUCCIÓN}

Los bosques tropicales son uno de los ecosistemas más diversos sobre el planeta, además de proporcionar diversos servicios ecosistémicos (de Beenhouwer, Aerts, y Honay, 2013). Sin embargo, acciones como la extracción de recursos, los asentamientos humanos, el exceso de residuos y la modificación de grandes áreas de producción (sistemas agroforestales, monocultivos, pastos para ganadería, etc.) degradan estos ecosistemas y ponen en peligro la biodiversidad que contienen (de Beenhouwer et al, 2013). Así, los ecosistemas pierden capacidad de recuperación y los servicios que prestan se ven amenazados (Andrade, 2011). La mayoría de esfuerzos por conservar esta biodiversidad, se han enfocado en aislar áreas relativamente bien conservadas de ecosistemas naturales bajo figuras de áreas protegidas (SánchezClavijo, Durán, Vélez, García, y Botero, 2010). Sin embargo, estas áreas naturales protegidas no son suficientes, ya que cubren menos del $15 \%$ de la superficie terrestre mundial (UNEP-WCMC y IUCN, 2016). Por consiguiente, es necesario complementar las áreas naturales protegidas con otras manejadas por principios ecológicos, que garanticen tanto la autosuficiencia como la producción de recursos, así como la conservación de la biodiversidad (Moguel y Toledo, 1999; Zermeño-Hernández, Pingarroni, y Martínez-Ramos, 2016).

Los sistemas agroforestales se consideran una alternativa o complemento para amortiguar la pérdida de biodiversidad, ya que son cultivos agrícolas con un manejo intencional de árboles de sombra, siendo importantes puesto que no remueven la totalidad de árboles del bosque y conservan parches de bosque nativo. Estos sistemas pueden aliviar la presión sobre el uso de recursos en las áreas protegidas, aumentar la conectividad de los componentes del paisaje (Bhagwat, Willis, Birks, y Whittaker, 2008; Zermeño-Hernández et al, 2016) y además, por su estructura multiestratificada, pueden proporcionar hábitat (Hall, Krausman, y Morrison, 1997) y diversos recursos a una significativa diversidad de animales y plantas, dependiendo del mantenimiento de la cubierta forestal y la intensificación del uso de la tierra (Cassano, Barlow, y Pardini, 2012; Guiracocha, Harvey, Somarriba, Krauss, y Carrillo, 2001).

Uno de los cultivos desarrollados bajo esta modalidad es el de cacao, Theobroma cacao L.; en Colombia, el cacao tiene un área total sembrada de 176.050 ha, cultivándose en suelos con características de piso térmico cálido, que comprende la franja de tierras ubicadas desde el nivel del mar hasta los $1300 \mathrm{~m}$ s. n. m. El departamento que concentra la mayor producción es Santander con el 41,45\% de participación, lo que equivale a 23.574 toneladas. Le siguen en importancia, con menor participación: Antioquia, Arauca, Huila, Tolima, Nariño y Cesar, los cuales, en conjunto representan el 40,85\% del total nacional (Federación Nacional de Cacaoteros, 2018).

Debido a la gran área que abarcan estos cultivos, su proximidad con la región Andina y su alta biodiversidad, son inevitables los encuentros con la fauna silvestre (ungulados, primates, roedores, aves granívoras, entre otros) que persiste en estas zonas. Eventualmente, algunas de estas especies pueden ocasionar daños a los cultivos o entrar en conflicto con las personas y poblaciones que dependen de dichos cultivos como sus ingresos económicos primarios (Conover, 2001; Marchini, 2014; Sillero-Zubiri, Sukumar, y Treves, 2007).

La fauna silvestre, que cohabita con los agroecosistemas, puede generar problemas con los productores por el eventual consumo y destrucción de cultivos 
y alimentos almacenados o la transmisión de enfermedades a humanos $\mathrm{u}$ otros animales, entre otras interacciones (Sillero-Zubiri et al, 2007; Treves, Wallace, y White, 2009). Estudios previos han registrado casos de conflictos con fauna y evaluado el daño hecho por mamíferos en plantaciones. Por mencionar algunos, Hafidzi (1998) reportó ratones y ardillas como las mayores plagas para los cultivos de cacao en Malasia, realizando un estudio sobre patrones de actividad de ardillas en estos cultivos, para proponer estrategias de manejo. Mollineau, Bekele, y García (2008) reportan que, en plantaciones de cacao a nivel industrial, en Trinidad, la ardilla roja (Notosciurus granatensis) tiene una marcada preferencia por los frutos maduros, dejando cuantiosas pérdidas, ya que hasta los frutos ligeramente afectados se pierden debido a la posterior acción de hongos. También indicaron que las zonas más afectadas son los bordes de los cultivos, puesto que están próximos a áreas boscosas, lo que permite su movimiento entre las zonas de alimentación (los cultivos) y sus refugios (los bosques). Por lo tanto, el manejo de estos encuentros (conflictos), con la fauna silvestre se aborda desde diferentes perspectivas, resultando en diversas respuestas por parte de las especies nativas (Muñoz y Muñoz-Santibáñez 2016).

En Colombia, los estudios sobre fauna en sistemas agroforestales, específicamente sobre mamíferos no voladores asociados a estos sistemas son limitados y tampoco son muchos los que registran las afectaciones hechas por estas especies a los cultivos. Por ejemplo, una evaluación de la riqueza y abundancia relativa de mamíferos no voladores habitando un cultivo de palma africana, en el departamento de Casanare, encontró un total de 16 especies, donde la mayoría correspondían a mesodepredadores y generalistas (Pardo-Vargas y Payán, 2015). Sin embargo, estudios enfocados en ensamblajes de murciélagos asociados a cultivos de café (Castaño, Botero, Velásquez, y Corrales, 2004; Numa, Verdu, y Sánchez-Palomino, 2005; Ortegón-Martínez y PérezTorres, 2007), registran la abundancia de especies como Glossophaga soricina, Sturnira lilium, Artibeus lituratus y Carollia brevicauda; concluyendo que los cafetales con sombrío y las coberturas de bosque nativo (parches) constituyen una fuente importante de alimento y refugio para algunos murciélagos frugí- voros y nectarívoros. Otros estudios en sistemas agroproductivos (Botero-Echeverri, Lentijo-Jiménez, y Sánchez-Clavijo, 2014; Sánchez-Clavijo et al, 2008, 2010), aunque no se enfocan en mamíferos, cobran importancia pues incluyen estudios donde se evalúa la biodiversidad asociada a cultivos de café en el país, enfocándose en aves, plantas y hormigas. Observaciones ocasionales también han mostrado algunos mamíferos involucrados en el daño a plantaciones de pino (Pinus patula) en el oriente de Antioquia (S. Solari, obs. pers.). Sin embargo, no se encontraron reportes publicados que abarquen la fauna asociada a cultivos de cacao realizados en Colombia.

Históricamente, las soluciones al conflicto con la fauna han sido letales, mediante cacería, envenenamiento, trampas o exclusión completa (Treves y Naughton-Treves, 2005). Sin embargo, los mamíferos considerados como potenciales plagas deben ser vistos, además, en términos de sus interrelaciones con los componentes del ecosistema y los potenciales roles que desempeñan (Bhagwat et al, 2008; Jose, 2009; Lacher et al, 2019; Rumiz, 2010). Algunos de estos roles ecológicos incluyen: influir en la composición vegetal del bosque (al depredar semillas y plántulas), en las propiedades químicas y físicas del suelo (aireación al excavar madrigueras), en la productividad primaria (consumo de hojas y frutos); en el reciclaje de nutrientes (carroñeros); al proporcionar fuente de alimento base para carnívoros o el control de insectos y fauna menor, entre otras (Aguilera, 1985; Rumiz, 2010; Sieg, 1987; Solari, Rodríguez, Vivar, y Velazco, 2002; Voss, 1991). Todo lo anterior contribuye a la integridad de los ecosistemas y al flujo de materia y energía entre los diferentes ambientes (Feldhamer, Drickamer, Vessey, Merritt, y Krajewski, 2007; NagyReis, Ribeiro, Setz, y Chiarello, 2019; Norris, 2012; Rojas y Moreno, 2014). Por todo lo anterior, la alteración de las comunidades de mamíferos, a través de programas de control (exclusión y cacería), influye en otros componentes y, finalmente, sobre todo el sistema (Sieg, 1987). Para contrarrestarlo, se ha generado un interés por alternativas a las represalias, como el manejo no letal de fauna, elevando la tolerancia para la vida silvestre (Treves et al, 2009), que permita el funcionamiento de estas redes de interacción y el desempeño de sus roles ecológicos. Una visión integral al tema de los conflictos con fauna 
silvestre pasa necesariamente por entender estos roles a escala local y regional (Nagy-Reis et al, 2019), lo que puede generar mayor tolerancia a la presencia de estas especies y un manejo racional ambientalmente.

La Compañía Nacional de Chocolates ha mostrado interés en saber cuáles mamíferos no voladores persisten en la Granja Yariguíes, ubicada en el departamento de Santander, ya que al incluir parches de bosque natural como zonas de reserva alrededor de los cultivos experimentales de cacao, se estaría favoreciendo la permanencia de estos animales. Por otro lado, también existe el interés en cuantificar los daños causados a las mazorcas de cacao, principalmente por la ardilla roja ( $N$. granatensis) e identificar qué otras especies pueden estar causando afectaciones negativas. En este sentido, el objetivo de este estudio fue identificar los roles ecológicos suministrados por mamíferos no voladores en un sistema agro-productivo de cacao, incluyendo los posibles daños que algunas especies ejercen sobre el cultivo.

\section{MATERIALES Y MÉTODOS}

\section{Lugar de estudio}

El lugar de muestreo fue la Granja Yariguíes, propiedad de la Compañía Nacional de Chocolates, ubicada en la vereda La Lejía, entre los municipios de San Vicente del Chucurí y Barrancabermeja, Santander. Con una extensión de 263 ha, donde 210 ha son destinadas al cultivo de cacao, mientras que 53 ha están destinadas a áreas de reserva y protección. La formación predominante (zona de vida) es bosque húmedo tropical (Hernández-Camacho, Hurtado, Ortiz, y Walschburger, 1992). La granja es atravesada por el río Oponcito y está sobre la vía Ruta del Sol (figura 1). La zona de cultivo de cacao está distribuida en 39 lotes, de los cuales se escogieron dos áreas que cumplieran las siguientes condiciones: (1) que tuvieran borde colindante con el bosque de reserva; lote 10, con un área de 7,3 ha, y (2) que fueran netamente zona de cultivo; lotes 5 y 6 , con un área de 3,5 ha y 3 ha, respectivamente, sumando un total de 6,5 ha. Se escogieron estos dos lotes juntos con el fin de tener áreas comparables, en cuanto al tamaño, con la parcela 10 (figura 1).

\section{Diseño de muestreo}

Los muestreos comprendieron cinco salidas de campo (una por mes) con una duración aproximada de cinco días cada una, desde septiembre de 2017 a enero de 2018. Los muestreos se dividieron en identificación y cuantificación de daños a las mazorcas (septiembre diciembre 2017) y registro de mamíferos (septiembre 2017 - enero 2018). En cada uno de los lotes se hicieron cinco recorridos lineales de $200 \mathrm{~m}$ de longitud (2 m a cada lado), ubicando los árboles con frutos afectados, para luego identificar y cuantificar los daños causados por roedores silvestres (ardillas, ñeques, puercoespines). Adicionalmente, estos recorridos permitieron identificar los lugares más afectados y evaluar si había alguna preferencia por el estado de maduración (inmaduro, medio o maduro) de los frutos. Los daños a las mazorcas se determinaron por observación directa, mientras que su cuantificación se calculó a partir del número de frutos afectados (mordidos o consumidos) por cada lote, así como mediante el porcentaje de estos respecto al total disponible (por lote). Finalmente, la identificación de la especie causante se hizo mediante comparación directa de las marcas de los incisivos con la dentición de especímenes depositados en la Colección Teriológica de la Universidad de Antioquia (CTUA).

Para determinar la presencia de los mamíferos que habitan la zona, se hicieron recorridos que permitieron encontrar rastros como huellas, madrigueras, excretas, entre otros (Aranda, 2012; Navarro y Muñoz, 2000), así como áreas de presencia potencial de especies menores, usualmente con buena cobertura original (bosque húmedo). Para obtener registros directos, se instalaron cuatro cámaras de detección automática (cámaras trampa), en lugares donde se consideró la potencial presencia de mamíferos ( $\sin$ usar atrayentes), las cuales se cambiaron de lugar cada mes para abarcar nuevos lugares que permitieran obtener más registros. También se dispusieron 40 trampas de captura viva, Sherman ${ }^{\circledR}(23 \mathrm{~cm})$, con el fin de obtener registros de pequeños mamíferos, que se ubicaron en líneas a través del borde del bosque o cerca a quebradas, donde se esperaba la presencia de estas especies, separadas entre sí por aproximadamente $10 \mathrm{~m}$. Se usó una mezcla de hojuelas de avena y mantequilla de maní como cebo. 


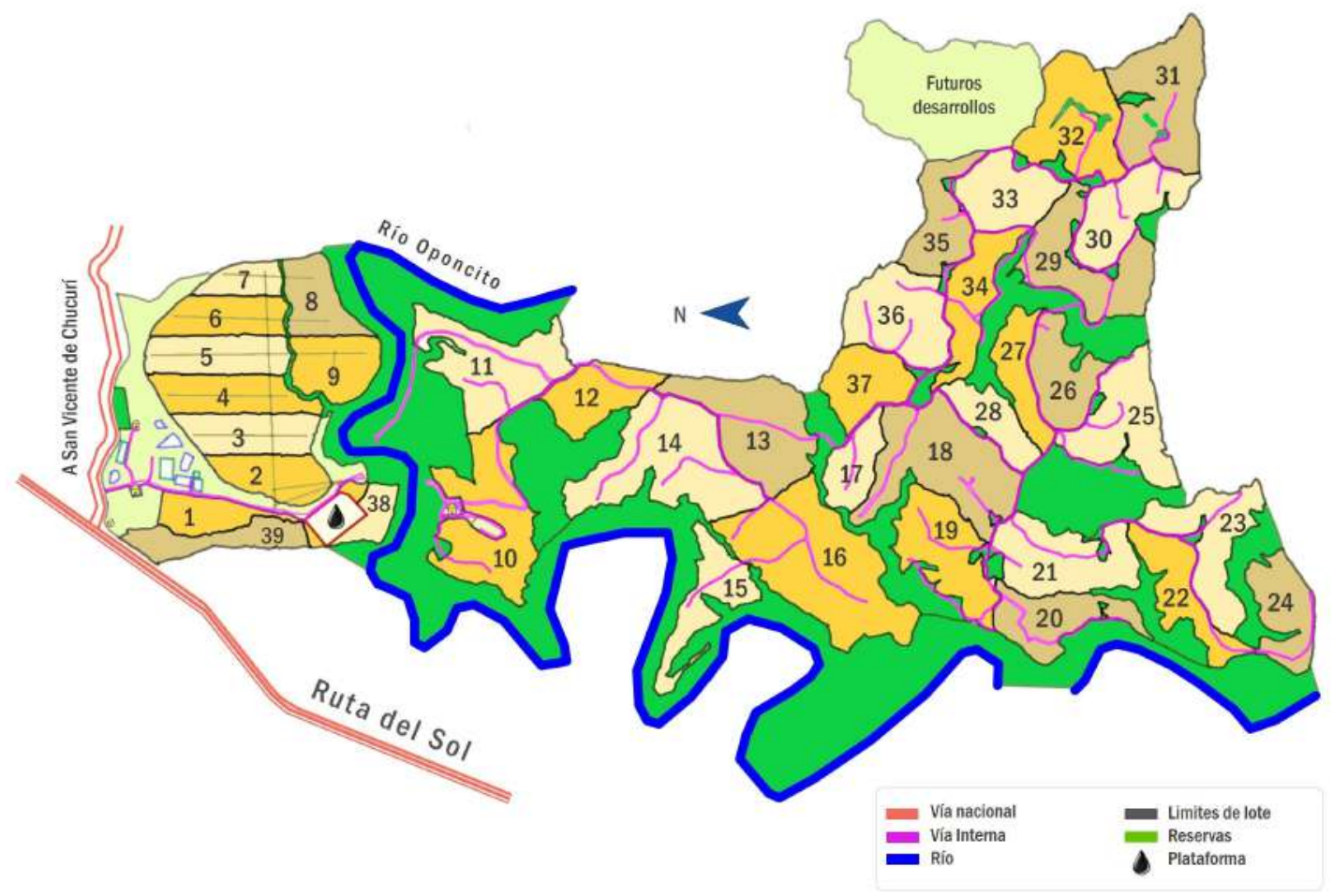

Figura 1. Mapa esquemático de la Granja Yariguíes, Santander, mostrando la ubicación de las parcelas experimentales de cacao (numeradas) y el bosque de protección.

Por otro lado, se encuestaron algunos colaboradores de la granja, incluyendo técnicos (4), personal de vigilancia (2) y supervisores (1), acerca de la presencia de los mamíferos que habitan la zona, ya que ellos tienen un conocimiento más amplio del lugar y podrían dar información sobre las especies que ven frecuentemente y los lugares que transitan. Para una mayor precisión en las identificaciones, se empleó el apoyo visual con dibujos en guías de mamíferos neotropicales (Eisenberg, 1989; Emmons y Feer, 1990).

El esfuerzo de muestreo se definió de diferentes formas según el método empleado; para las cámaras trampa se definió como la suma del número de horas que las cámaras estuvieron activas multiplicado por el número de cámaras utilizadas. Para las trampas Sherman ${ }^{\circledR}$, se definió como el número de noches que las trampas estuvieron activas multiplicado por el número de trampas (Voss y Emmons, 1996), mien- tras que para los recorridos libres como la suma del tiempo invertido por día muestreado (Pardo-Vargas y Payán-Garrido, 2015).

\section{Roles ecológicos}

Estos mamíferos, además de la posible afectación a los cultivos, también juegan un rol particular dentro del ecosistema estudiado, siendo necesario establecer cuáles son estas especies y con cuáles otras coexisten en el lugar de estudio. Una vez identificados taxonómicamente se estableció, mediante la revisión de literatura pertinente, sus roles ecológicos en este ambiente. Para determinar estos roles, las especies se dispusieron por gremios, término definido por Root (1967), el cual agrupa las especies que explotan la misma clase de recursos ambientales de una manera similar sin tener en cuenta la posición taxonómica. Para esto se tomó en cuenta la dieta de cada especie 
registrada durante este estudio, así como el sustrato utilizado por cada una y el horario predominante de su actividad (González-Salazar, Martínez-Meyer, y López-Santiago, 2014):

Dieta: para clasificar las especies por su dieta se consideró el principal recurso alimenticio utilizado por los mamíferos encontrados como: vertebrados e invertebrados no-artrópodos (carnívoros), insectos (insectívoros), carroña (carroñeros), frutos (frugívoros), hojas, tallos o semillas (herbívoros). Sustrato de forrajeo: se refiere al lugar donde los organismos obtienen el alimento. Se consideraron tres sustratos principales: el suelo, los árboles y los cuerpos de agua, donde se desempeñan como cazadores, recolectores y excavadores, entre otros. Periodo de actividad: se dividió en dos clases: 1) Diurna: si el periodo de actividad se inició en la mañana y continuó durante el día (06:00 a 18:00 h). 2) Nocturna: si la actividad comenzó a altas horas de la tarde y continuó durante toda la noche (18:00 a 06:00 h).

Para asignar las especies a un gremio se consideraron todos los datos obtenidos, ya sea por observación durante el muestreo o por información obtenida de la literatura. Por otro lado, en los casos que no se pudo determinar el tipo de dieta para la especie, se consideró la información reportada para el género (González-Salazar et al, 2014).

Para evaluar las diferencias entre las especies en relación con los daños causados al cacao, así como entre la actividad de la ardilla entre parcelas y entre meses, utilizamos una prueba de Kruskal-Wallis $(p=0,05)$ dado que los valores obtenidos no cumplieron los supuestos requeridos para una prueba de ANOVA (McDonald, 2014).

\section{RESULTADOS}

\section{Daños en los cultivos}

Se registró a la ardilla roja ( $N$. granatensis) como la especie que más atacó las mazorcas de cacao (Kruskal-Wallis $\mathrm{H}=8,3434 ; \mathrm{gl}=2 ; p=0,015$ ), seguida del ñeque (Dasyprocta punctata) y del puercoespín (Coendou sp.), aunque estos dos últimos en una muy baja proporción (tabla 1). La incidencia de daño en los lotes 5-6 fue muy baja y el porcentaje de daño no varió mucho entre muestreos (0 a 0,34\%), excepto por septiembre, que mostró un porcentaje más alto $(3,2 \%)$; un patrón similar se observó en el lote 10 (figura 3). Sin embargo, cuando se combinaron ambos lotes, el número de frutos afectados en septiembre (86) no fue muy distinto al registrado en el mes de octubre (84), lo que evidencia que, en general, hay un comportamiento similar en estos dos meses. En general, se observó un decrecimiento en el número de frutos y el porcentaje de daño a medida que pasa el tiempo (tabla 1), pero que no llega a ser significativo $(\mathrm{H}=3 ; \mathrm{gl}=3 ; p=0,3916)$. También se pudo identificar que la zona más afectada fue el lote 10 (figura 2), aunque las diferencias no resultaron significativas $(\mathrm{H}=2.1084 ; \mathrm{gl}=1 ; p=0,1465)$.

En cuanto a la preferencia por algún estado de madurez del fruto, se evidenció una fuerte predilección por los frutos maduros, incluyendo en promedio más del $50 \%$ de los frutos afectados (tabla 2). Sin

Tabla 1. Número de frutos afectados por ardilla roja (Notosciurus granatensis), ñeque (Dasyprocta punctata) y puercoespín (Coendou sp.) en cada lote por mes de muestreo. Se observa la mayor incidencia de las ardillas en el lote 10, mientras que los ñeques y puercoespines no representan mayor daño. Para los lotes 5-6, los daños fueron comparativamente más bajos que los del lote 10.

\begin{tabular}{|c|c|c|c|c|c|c|}
\hline & \multicolumn{6}{|c|}{ Especie causante de daños } \\
\hline \multirow[t]{2}{*}{ Mes } & \multicolumn{3}{|c|}{ Parcela 5-6 } & \multicolumn{3}{|c|}{ Parcela 10} \\
\hline & Ardilla & Ñeque & Puercoespín & Ardilla & Ñeque & Puercoespín \\
\hline Septiembre & 3 & 0 & 0 & 81 & 2 & 0 \\
\hline Octubre & 5 & 0 & 0 & 79 & 0 & 0 \\
\hline Noviembre & 0 & 0 & 0 & 43 & 0 & 1 \\
\hline Diciembre & 3 & 0 & 0 & 12 & 2 & 0 \\
\hline Total & 11 & 0 & 0 & 215 & 4 & 1 \\
\hline
\end{tabular}


embargo, no se encontraron diferencias significativas entre las tres categorías definidas $(\mathrm{H}=5,258 ; \mathrm{gl}=2$; $p=0,0715)$.

\section{Registros de mamíferos}

Se registró un total de 17 especies en cinco órdenes empleando los diferentes métodos antes mencionados (cámara-trampa, rastreo de huellas, rastreo de madrigueras, trampas Sherman ${ }^{\circledR}$, entre otros). Como se esperaba, la mayoría de los registros se dieron en el bosque de protección (tabla 3). Mediante conversaciones con el personal de la granja se confirmaron algunos registros que inicialmente se tomaron como potenciales (v.g., nutria, chigüiro) ya que la eviden- cia no era concluyente. En la mayoría de casos fue posible determinar con precisión la especie a partir de las fotografías o videos, o cuando se tuvo acceso a algún material (ejemplares en trampas, restos); solo en el caso del puercoespín (Coendou sp.), identificado a partir del tamaño de la huella de los incisivos en las mazorcas, fue imposible establecer la identidad de la especie.

\section{Representatividad de Gremios}

Dado que la definición de gremio usada por nosotros incluye grupo alimenticio, sustrato y horario de actividad, las 17 especies registradas se incluyeron en 13 gremios que representan cinco grupos alimenti-

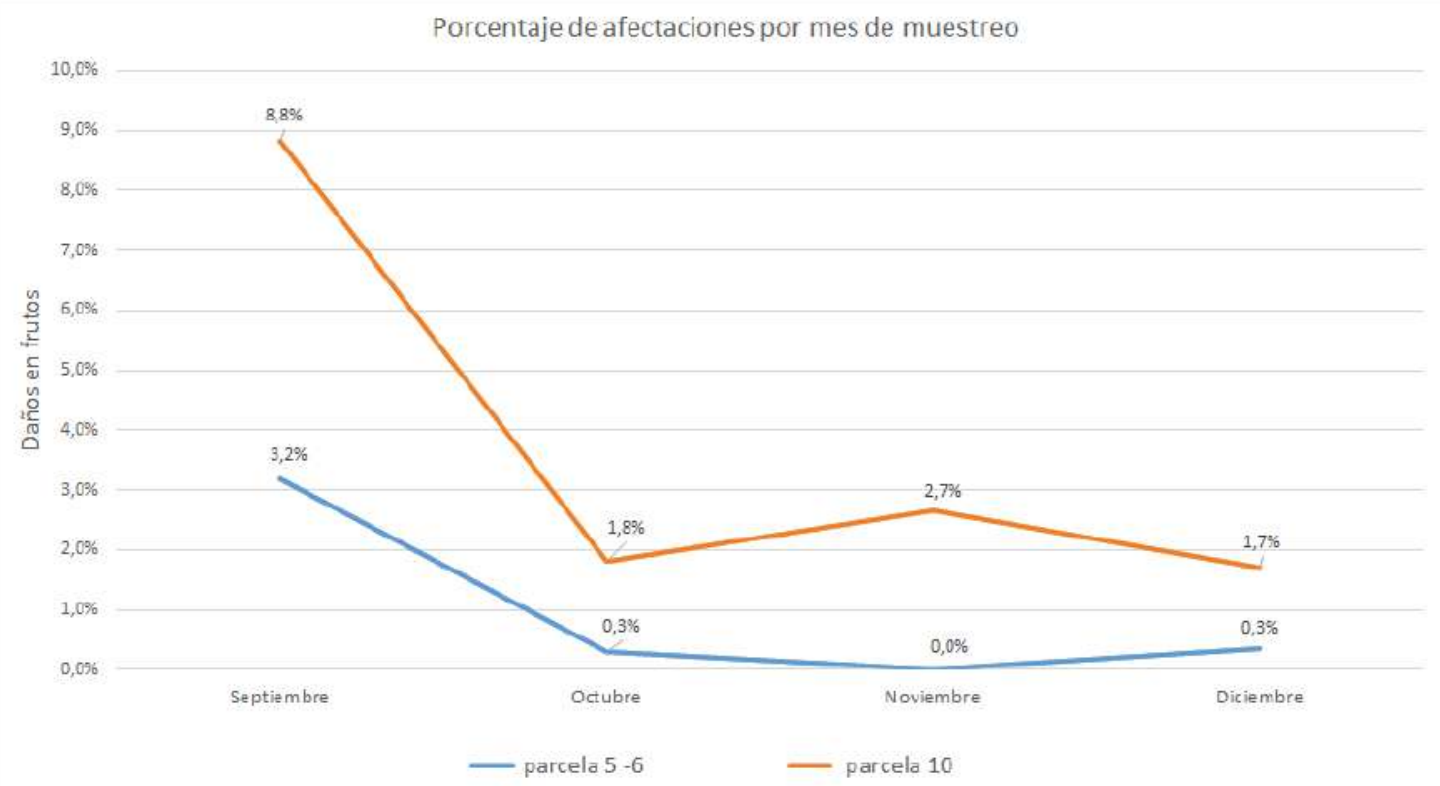

Figura 2. Porcentajes de afectaciones en frutos por mes de muestreo. La línea azul representa los daños para los lotes 5-6; la línea roja representa los daños para el lote 10.

Tabla 2. Frutos afectados por grado de madurez en cada lote durante los meses de muestreo, donde se observa una clara preferencia por los frutos maduros.

\begin{tabular}{|c|c|c|c|c|c|c|}
\hline & \multicolumn{6}{|c|}{ Afectación según maduración del fruto } \\
\hline \multirow[t]{2}{*}{ Mes } & \multicolumn{3}{|c|}{ Parcela 5-6 } & \multicolumn{3}{|c|}{ Parcela 10} \\
\hline & Maduro & Medio & Inmaduro & Maduro & Medio & Inmaduro \\
\hline Septiembre & 2 & 1 & 0 & 51 & 10 & 22 \\
\hline Octubre & 3 & 2 & 0 & 54 & 14 & 11 \\
\hline Noviembre & 0 & 0 & 0 & 29 & 15 & 0 \\
\hline Diciembre & 3 & 0 & 0 & 10 & 4 & 0 \\
\hline Total & 8 & 3 & 0 & 144 & 47 & 33 \\
\hline
\end{tabular}


Tabla 3. Listado de mamíferos registrados en la Granja Yariguíes, donde se observa los meses en los cuales fueron registrados cada uno y el lugar donde se obtuvo el registro. Las letras B, C, R, corresponden a los lugares de muestreo Bosque, Cultivo, y Río respectivamente. * Indica registros hechos por fuera de estas fechas, por personal de la granja o por subsecuentes visitas de los investigadores (en Bosque).

\begin{tabular}{|c|c|c|c|c|c|c|}
\hline & \multicolumn{6}{|c|}{ Mes de muestreo } \\
\hline Especie & Nombre común & Septiembre & Octubre & Noviembre & Diciembre & Enero \\
\hline Dasypus cf. novemcinctus & Armadillo & $B, C$ & $\mathrm{~B}, \mathrm{C}$ & $\mathrm{B}, \mathrm{C}$ & $B$ & $B$ \\
\hline Didelphis marsupialis* & Zarigüeya & & & & & B \\
\hline Metachirus nudicaudatus* & $\begin{array}{l}\text { Chucha } \\
\text { mantequera }\end{array}$ & & & & & \\
\hline Marmosa robinsoni & Marmosa & & & & & B \\
\hline Tamandua tetradactyla* & Oso mielero & & & & & \\
\hline Bradypus variegatus* & Oso perezoso & & & & & \\
\hline Cuniculus paca & Guagua & $B, C$ & B & $B, R$ & $B, R$ & $B, R$ \\
\hline Hydrochoerus hydrochaeris & Chigüiro & $\mathrm{R}$ & $\mathrm{R}$ & $\mathrm{R}$ & $\mathrm{R}$ & $\mathrm{R}$ \\
\hline Notosciurus granatensis & Ardilla roja & $B, C$ & $B, C$ & $B, C$ & $B, C$ & $B, C$ \\
\hline Coendou sp. & Puercoespín & & & C & & \\
\hline Dasyprocta punctata & Neque & C & & & C & \\
\hline Proechimys cf. chrysaeolus & Rata espinosa & C & & B & & B \\
\hline Leopardus pardalis & Tigrillo & B & C & C & & \\
\hline Lontra longicaudis & Nutria & & R & & & \\
\hline Procyon cancrivorus & Mapache & B & $B, R$ & $B, R$ & $B, C, R$ & $B, C, R$ \\
\hline Cerdocyon thous & Zorro perro & $B, C, R$ & $B, C, R$ & $B, R$ & $B, R$ & $B, R$ \\
\hline Eira barbara & Tayra & & & B & B & \\
\hline
\end{tabular}

cios (insectívoros, carnívoros, herbívoros, frugívoros y omnívoros). En los casos en que no fue posible definir el sustrato, dado que algunas especies son capaces de utilizar distintos estratos (suelo y vegetación arbórea), solo se indicó el grupo alimenticio y el patrón de actividad (tabla 4).

\section{DISCUSIÓN}

Dado que en la granja Yariguíes la cobertura original de las zonas de bosque, aunque fragmentadas, se ha mantenido sin mayores cambios durante los últimos 20 años, consideramos que éstas siguen proporcionando hábitats favorables y recursos para las especies nativas que persisten en la zona. Las zonas de cultivo, por otro lado, también son aprovechadas directamente para alimentación por animales como ardillas y ñeques, así como indirectamente (como corredores para tránsito) por armadillos, mapaches y zorros, entre otros. Sin embargo, no se evidencia una oferta de refugios o hábitats favorables para estas especies en la zona de cultivo, a causa de podas continuas, poca diversidad de plantas y el paso constante de trabajadores, entre otros factores. El avistamiento de diferentes evidencias en zonas de cultivo (como huellas de zorro, mapache, tigrillo y guagua, entre otros) sugiere que estas zonas proporcionan, principalmente, conectividad entre los parches de bosque (Beer et al, 2003). De esta manera, el mantenimiento de los parches de bosque con características similares a ambientes poco intervenidos estaría ayudando a atenuar los efectos de la intensificación agrícola al proporcionar hábitats para poblaciones de mamíferos, contribuyendo al mantenimiento de la biodiversidad y las redes tróficas en estos ecosistemas modificados (Gómez, Coda, Serafini, Steinmann, y Priotto, 2017).

La presencia de especies generalistas de hábitat (sensu Rozenweig, 1981), tales como zorros, mapaches, zarigüeyas y armadillos eran esperadas puesto que estas poblaciones toleran mejor los cambios ambientales (Abba, Zufiaurre, Codesido, y Bilenca, 2015; Bhagwat et al, 2008; Gómez et al, 2017). Pero, la presencia de especies de hábitos carnívoros, como tigrillos y nutrias, que requieren condiciones específicas de hábitat debido a sus dietas y comportamiento, sugiere que al menos durante el estudio se dieron condiciones aceptables en los parches de bosque que 
Tabla 4. Especies registradas en la Granja Yariguíes, agrupadas por grupos tróficos y hábitos (tipo de sustrato y patrón de actividad).

\begin{tabular}{|c|c|c|c|c|}
\hline Grupo trófico & Hábito & Orden & Familia & Especie \\
\hline \multirow[b]{2}{*}{ Insectívoro } & Terrestre nocturno & Cingulata & Dasypodidae & Dasypus cf. novemcinctus \\
\hline & Arbóreo nocturno & $\begin{array}{l}\text { Pilosa } \\
\text { Didelphimorphia }\end{array}$ & $\begin{array}{l}\text { Myrmecophagidae } \\
\text { Didelphidae }\end{array}$ & $\begin{array}{l}\text { Tamandua tetradactyla } \\
\text { Marmosa robinsoni }\end{array}$ \\
\hline \multirow{2}{*}{ Herbívoro } & Arbóreo nocturno & Pilosa & Bradypodidae & Bradypus variegatus \\
\hline & Acuático nocturno & Rodentia & Caviidae & Hydrochoerus hydrochaeris \\
\hline \multirow{4}{*}{ Frugívoro } & Terrestre diurno & Rodentia & Dasyproctidae & Dasyprocta punctata \\
\hline & Terrestre nocturno & $\begin{array}{l}\text { Rodentia } \\
\text { Rodentia }\end{array}$ & $\begin{array}{l}\text { Cuniculidae } \\
\text { Echimyidae }\end{array}$ & $\begin{array}{l}\text { Cuniculus paca } \\
\text { Proechimys cf. chrysaeolus }\end{array}$ \\
\hline & Arbóreo diurno & Rodentia & Sciuridae & Notosciurus granatensis \\
\hline & Arbóreo nocturno & Rodentia & Erethizontidae & Coendou sp. \\
\hline \multirow{2}{*}{ Carnívoro } & Terrestre nocturno & Carnivora & Felidae & Leopardus pardalis \\
\hline & Acuático diurno & Carnivora & Mustelidae & Lontra longicaudis \\
\hline \multirow{3}{*}{ Omnívoro } & Diurno & Carnivora & Mustelidae & Eira barbara \\
\hline & Terrestre nocturno & $\begin{array}{l}\text { Carnivora } \\
\text { Carnivora }\end{array}$ & $\begin{array}{l}\text { Procyonidae } \\
\text { Canidae }\end{array}$ & $\begin{array}{l}\text { Procyon cancrivorus } \\
\text { Cerdocyon thous }\end{array}$ \\
\hline & Nocturno & $\begin{array}{l}\text { Didelphimorphia } \\
\text { Didelphimorphia }\end{array}$ & $\begin{array}{l}\text { Didelphidae } \\
\text { Didelphidae }\end{array}$ & $\begin{array}{l}\text { Didelphis marsupialis } \\
\text { Metachirus nudicaudatus }\end{array}$ \\
\hline
\end{tabular}

permitieron la presencia de estas especies (figura 3). Igualmente, esto podría tomarse como un posible indicador de las buenas prácticas de manejo que se llevan a cabo en la granja. Aunque estos gremios tróficos no son equivalentes a grupos funcionales, posibles de asociar a ciertos servicios ecosistémicos (Blondel, 2003), esta caracterización permite un primer acercamiento a la estructura del ensamblaje de mamíferos (Rumiz, 2010) en una zona productiva y transformada, incluyendo ciertos procesos a escala del ecosistema y cómo ciertas especies podrían estar cumpliendo roles similares y complementarios (Lacher et al, 2019).

En cuanto a los daños en los cultivos, en los lotes 56 se registraron pocos daños (máximo cinco frutos por mes muestreado), mientras que el lote 10 tuvo los mayores registros de daño (con un máximo de 83 frutos afectados y un mínimo de 14), sobre todo en las mazorcas ubicadas en las primeras líneas de cultivo y que limitan con el bosque. Es posible que esto se deba principalmente a esa cercanía, ya que, como se ha mostrado previamente, el bosque ofrece un espacio seguro para los mamíferos, de manera que éstos solo utilizan los cultivos para alimentación o conexión con otras zonas por esa conveniencia (de Beenhouwer et al, 2013; Mollineau et al, 2008). La progresiva disminución de las afectaciones podría deberse a las dinámicas de la finca, donde los meses de noviembre y diciembre son las de mayor cosecha, por lo que la disponibilidad de mazorcas se ve reducida y al mismo tiempo aumenta la presencia de personal a través de los sembríos.

En general, estos valores sugieren que los mamíferos no están representando grandes problemas ni pérdidas económicas importantes en estos cultivos. La baja incidencia puede darse por una moderada población de las especies que afectan los cultivos (ardillas, ñeques, puercoespines), que tal vez está siendo controlada por depredadores que persisten en la zona (como zorros y tigrillos), lo que es importante pues dicho equilibrio ayuda a mantener reguladas las poblaciones de potenciales plagas como los roedores (Nagy-Reis et al, 2019; Rumiz, 2010), evitando así algunos conflictos mayores (daños severos) que se pudieran tener con la fauna local. Sin embargo, la diversidad de pequeños y medianos mamíferos también ayuda a sostener otras funciones básicas del ecosistema, ya sea conectando parches de hábitats diferentes o similares mediante el transporte de materia orgánica, nutrientes y minerales a lo largo del paisaje (Lacher et al, 2019; Priotto, 2017; Rumiz, 2010).

En cuanto a los gremios identificados, estos representan roles ecológicos que, dependiendo del recurso que explotan y la forma en que lo hacen, pueden traer varios beneficios tanto para los bosques como para los cultivos (Lacher et al, 2019). Por ejemplo, las especies de hábitos insectívoros pueden ejercer control pobla- 


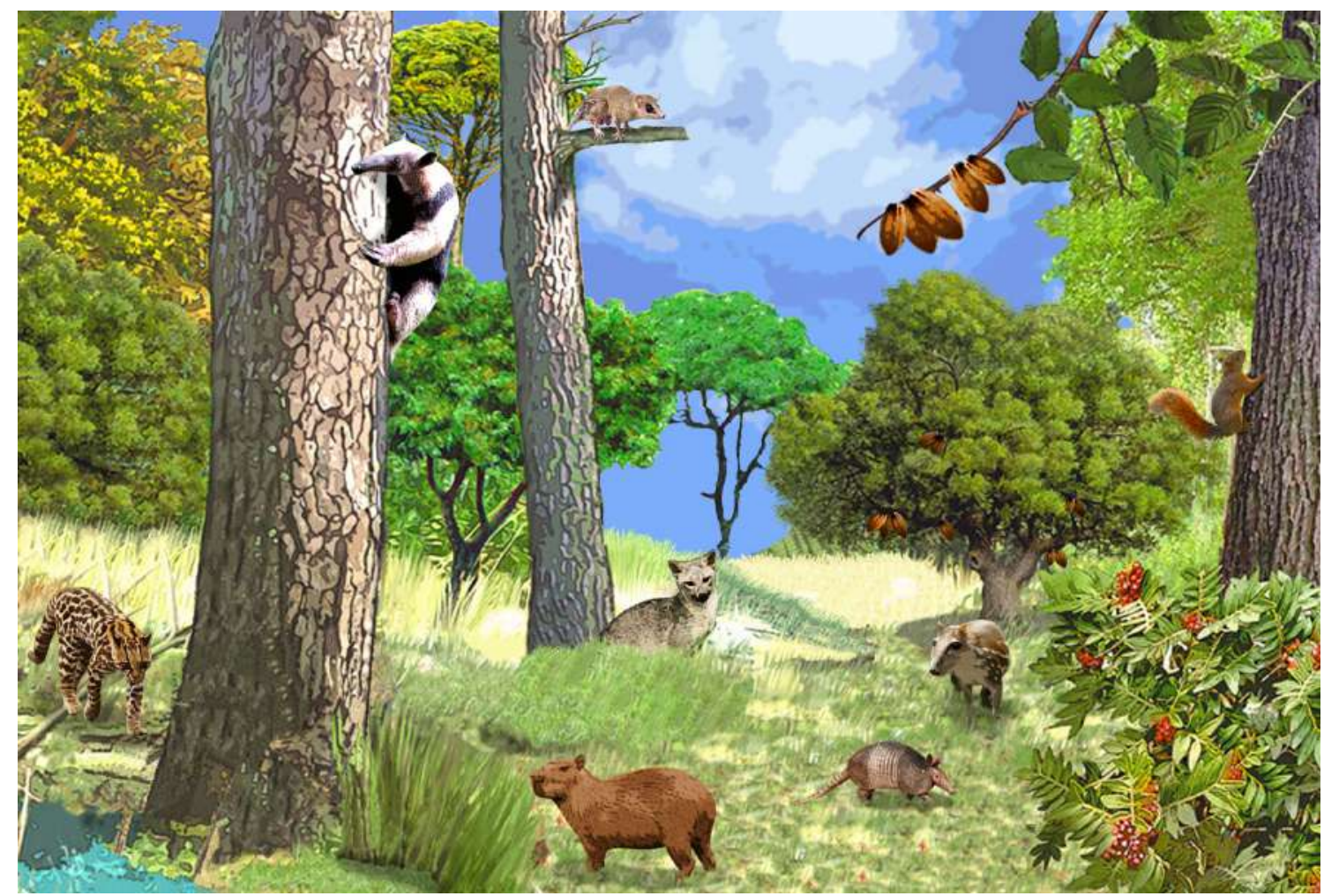

Figura 3. Representación del entorno estudiado en la Granja Yariguíes, mostrando algunas de las especies registradas. De izquierda a derecha: Ocelote (Leopardus pardalis; carnívoro terrestre nocturno), Tamandúa (Tamandua tetradactyla; insectívoro arbóreo nocturno), Marmosa (Marmosa robinsoni; omnívoro arbóreo nocturno), Chigüiro (Hydrochoerus hydrochaeris; herbívoro acuático nocturno), Zorro perro (Cerdocyon thous; omnívoro terrestre nocturno), Armadillo (Dasypus novemcinctus; insectívoro terrestre nocturno), Guagua (Cuniculus paca; frugívoro terrestre nocturno), Ardilla de cola roja (Notosciurus granatensis; frugívoro arbóreo diurno).

cional sobre insectos que afectan las plantas; mientras que, los armadillos al ser semifosoriales airean y remueven el suelo al hacer excavaciones continuas en busca de alimento o elaboración de madrigueras, lo que permite la aireación y remoción del suelo evitando la compactación (Abba et al, 2015; Bilenca et al, 2017; Rumiz, 2010). De otro lado, animales como tigrillos (carnívoros), zorros, mapaches y tayras (omnívoros), pueden estar influenciando las poblaciones de pequeños mamíferos que pueden ejercer daños en los cultivos (Nagy-Reis et al, 2019) o que pueden afectar el ecosistema si sus poblaciones aumentan considerablemente, por ejemplo, consumiendo semillas y plántulas que podrían perjudicar la dispersión y por ende, la composición del bosque (Rojas-Robles, Stiles, y Muñoz-Saba, 2012). Igualmente, herbívoros como el chigüiro y la guagua se alimentan sobre la tierra y defecan en el agua, favoreciendo el enriquecimiento de los ambientes acuáticos con materia orgánica y semillas, de lo cual se beneficiarían los peces y otros organismos (Beck, von Helversen, y Beck-King, 1999). Adicionalmente, para las especies que explotan los mismos recursos, es posible reducir la competencia directa (p. ej., por alimentos, en caso de su escasez) ya sea utilizando un estrato o un horario de actividad diferente (González-Salazar et al, 2014).

Debe recordarse también que, roedores como las ardillas, ñeques y guaguas (gremio de frugívoros), también influyen en la composición de los bosques, ya que la depredación de los frutos permite el transporte de semillas (Lacher et al, 2019; Rumiz, 2010). Los frugívoros consumen los frutos cerca de la planta 
parental y en algunos casos alejan y entierran semillas para consumo posterior, las cuales en gran parte son olvidadas, lo que permite la germinación de las plantulas, aumentando las áreas ocupadas por estas y sus rangos de distribución (Rojas-Robles et al, 2012). Esto cobra importancia teniendo en cuenta que en los bosques tropicales, más del $75 \%$ de las especies leñosas dependen de animales para dispersar sus semillas (Janzen y Vázquez-Yanes, 1991). Estas especies, junto con otras como el puercoespín (Coendou sp.), pueden estar actuando directamente sobre la regeneración natural del bosque ya que, al ramonear algunas especies de plantas de rápido crecimiento, ayudan a reducir la capacidad competitiva de estas, favoreciendo el crecimiento de plantas con más lento crecimiento (Lacher et al, 2019; Rumiz, 2010).

Por último, cabe resaltar que la Compañía Nacional de Chocolates no hace control o manejo de animales considerados "plagas", y por el contrario, al mantener una zona de reserva en esta granja ayuda con la preservación de estas poblaciones. El rol ecológico de las especies presentes en este ecosistema productivo, complementado con medidas relativas de su valor funcional como reguladores de otras poblaciones, y sobre la dinámica general del ecosistema, resulta altamente provechoso para clasificar y categorizar especies o grupos de especies, tanto con fines de investigación como de conservación (Lacher et al, 2019; Nagy-Reis et al, 2019). Por ello, este proceso resulta crítico para el mantenimiento de esta biodiversidad y su dinámica, y favorece un uso racional y adecuado de los servicios ecosistémicos asociados.

\section{AGRADECIMIENTOS}

Los autores agradecen al Grupo Mastozoología de la Universidad de Antioquia, por el apoyo con los equipos de campo y el financiamiento de las salidas de campo. Igualmente, a los colegas E. Patiño C., T. Villada C. y J. Daza Gallo, por el acompañamiento en algunos muestreos, y al personal de apoyo en la granja Yariguíes. También a L. M. López R. por diseñar y preparar la figura 3. Asimismo, agradecemos al área de Compras y Fomento Agrícola de la Compañía Nacional de Chocolates por permitir la ejecución de esta investigación en la Granja Yariguíes y por el acompañamiento en las salidas de campo. Los autores expresan su agradecimiento a dos evaluadores anónimos, quienes enriquecieron el manuscrito original con sus comentarios.

\section{CONFLICTO DE INTERESES}

Los autores declaran no tener conflictos de intereses.

\section{REFERENCIAS}

Abba, A. M., Zufiaurre, E., Codesido, M., y Bilenca, D. N. (2015). Burrowing activity by armadillos in agroecosystems of central Argentina: Biogeography, land use, and rainfall effects. Agriculture, Ecosystems and Environment, 200: 54-61. doi:10.1016/j.agee.2014.11.001

Aguilera, M. (1985). El estudio de los mamíferos en Venezuela: evaluación y perspectivas. Caracas, Venezuela: Asociación Venezolana para el Estudio de los Mamíferos AsoVEM y Fondo Editorial Acta Científica Venezolana.

Andrade, C. (2011). Estado del conocimiento de la Biodiversidad en Colombia y sus amenazas. Consideraciones para fortalecer la interacción Ciencia-Política. Revista de la Academia Colombiana de Ciencias Exactas, Físicas y Naturales, 35(137): 491-507.

Aranda Sánchez, J. M. (2012). Manual para el rastreo de mamíferos silvestres de México. México D.F.. México: Comisión Nacional para el Conocimiento y Uso de la Biodiversidad (CONABIO).

Beck, H., Von Helversen, O., y Beck-King, R. (1999). Home range, population density, and food resources of Agouti paca (Rodentia. Agoutidae) in Costa Rica: a study using alternative methods. Biotropica, 31(4): 675-685. doi:10.1111/j.1744-7429.1999.tb00417.x

Beer, J., Harvey, C., Ibrahim, M., Harmand, J. N., Somarriba, E., y Jiménez, F. (2003). Servicios ambientales de los sistemas agroforestales. Agroforestería en las Américas, 10(37-38): 80-87. http://hdl.handle.net/11554/6806

Bhagwat, S. A., Willis, K. J., Birks, H. J., y Whittaker, R. J. (2008). Agroforestry: a refuge for tropical biodiversity? Trends in Ecology and Evolution, 23(5): 261-267. doi:10.1016/j.tree.2008.01.005

Bilenca, D. N., Abba, A. M., Corriale, M. J., Pérez Carusi, L. C., Pedelacq, M. E., y Zufiaurre, E. (2017). De venados, armadillos y coipos: los mamíferos autóctonos frente a los cambios en el uso del suelo, los manejos agropecuarios y la presencia de nuevos elementos en el paisaje rural. Mastozoología Neotropical, 24(2): 277-287.

Blondel, J. (2003). Guilds or functional groups: does it matter? Oikos, 100: 223-231.

Botero-Echeverri, J. E., Lentijo-Jiménez, G. M., y SánchezClavijo, L. M. (2014). Biodiversidad en zonas cafeteras de Colombia. Principales lecciones. Chinchiná, Colombia: Cenicafé.

Cassano, C. R., Barlow, J., y Pardini, R. (2012). Large mammals in an agroforestry mosaic in the Brazilian Atlantic 
Forest. Biotropica, 44(6): 818-825. doi:10.1111/j.17447429.2012.00870.x

Castaño, J. H., Botero, J. B., Velásquez, S., y Corrales, J. D. (2004). Murciélagos en agroecosistemas cafeteros de Colombia. Chiroptera Neotropical, 10: 196-199.

Conover, M. R. (2001). Resolving human-wildlife conflicts: the science of wildlife damage management. Boca Raton, Estados Unidos: Lewis Publishers.

De Beenhouwer, M., Aerts, R., y Honnay, O. (2013). A global meta-analysis of the biodiversity and ecosystem service benefits of coffee and cacao agroforestry. Agriculture, Ecosystems and Environment, 175: 1-7. doi:10.1016/j.agee.2013.05.003

Eisenberg, J. F. (1989). Mammals of the Neotropics. The Northern Neotropics (Vol. 1). Panamá, Colombia, Venezuela, Guyana, Suriname, French Guiana. Chicago, Estados Unidos, y Londres, Reino Unido: University of Chicago Press.

Emmons, L. H., Feer, F. (1990). Neotropical rainforest mammals, a field guide. Chicago, Estados Unidos, $\mathrm{y}$ Londres, Reino Unido: University of Chicago Press. doi:10.1017/S0266467400005708

Federación Nacional de Cacaoteros. (2018). Economía Nacional. Recuperado el 29 de julio de 2019 de https://www.fedecacao.com.co/portal/index.php/es/ 2015-02-12-17-20-59/nacionales

Feldhamer, G. A., Drickamer, L. C., Vessey, S. H., Merritt, J. F., y Krajewski, C. (2007). Mammalogy: adaptation, diversity, ecology (4ta ed.). Baltimore, Estados Unidos: The Johns Hopkins University Press. doi:10.1086/690899

Gómez, M. D., Coda, J. A., Serafini, V. N., Steinmann, A. R., y Priotto, J. W. (2017). Small mammals in agroecosystems, responses to land use intensity and farming management. Mastozoología Neotropical, 24 (2): 289-300.

González-Salazar, C., Martínez-Meyer, E., y López-Santiago, G. (2014). A hierarchical classification of trophic guilds for North American birds and mammals. Revista Mexicana de Biodiversidad, 85(3): 931-941. doi:10.7550/rmb.38023

Guiracocha, G., Harvey, C. A., Somarriba, E., Krauss, U., y Carrillo, E. (2001). Conservación de la biodiversidad en sistemas agroforestales con cacao y banano en Talamanca, Costa Rica. Agroforestería en las Américas, 8: 7-11. http://hdl.handle.net/11554/5948

Hafidzi, M. N. (1998). Plantain Squirrel Callosciurus notatus in a plantation habitat. Pertanika Journal of Tropical Agricultural Science, 21(1): 23-28.

Hall, L. S., Krausman, P. R., y Morrison, M. L. (1997). The habitat concept and a plea for standard terminology. Wildlife Society Bulletin, 25: 173-182.

Hernández Camacho, J., Hurtado, A., Ortiz, R., y Walschburger, T. (1992). Unidades biogeográficas de Colombia. En: G. Halffter, M. E, Ramírez, R. Ramírez Chang, y M. Rebolledo. (Eds.). La Diversidad Biológica de Iberoamérica (pp. 105-151). México: Instituto de Ecología, A.C.

Janzen, D. H., y Vázquez-Yanes, C. (1991). Aspects of tropical seed ecology of relevance to management of tropical forested wildlands. En: A. Gómez-Pompa, T. C. Whitmore, y M. Hadley (Eds.). Rainforest regeneration and management. Man and the Biosphere Series 6 (pp. 137-
157). Francia: UNESCO y Parthenon Publishing Group.

Jose, S. (2009). Agroforestry for ecosystem services and environmental benefits: An overview. Agroforestry Systems, 76 (1): 1-10. doi:10.1007/s10457-009-9229-7

Lacher, T. E., Davidson, A. D., Fleming, T. H., Gómez-Ruiz, E. P., McCracken, G. F., Owen-Smith, N., Peres, C. A., y Vander Wall, S. B. (2019). The functional roles of mammals in ecosystems. Journal of Mammalogy, $100(3)$ : 942-964. doi:10.1093/jmammal/gyy183

McDonald, J. H. (2014). Handbook of Biological Statistics (3rd ed.). Baltimore, Estados Unidos: Sparky House Publishing.

Marchini, S. (2014). Who's in conflict with whom? Human dimensions of the conflicts involving wildlife. En: L. M, Verdade, M. C, Lyra-Jorge, y C. I, Piña (Eds.). Applied Ecology and Human Dimensions in Biological Conservation (pp. 189-209). Alemania: Berlin Heidelberg Springer-Verlag. doi:10.1007/978-3-642-54751-513

Moguel, P., y Toledo, V. M. (1999). Biodiversity conservation in tradicional Coffee systems of Mexico. Conservation Biology, 13(1): 11-21. doi:10.1046/j.1523-1739.1999.97153.x

Mollineau, W. M., Bekele, F., y García, G. W. (2008). The Neo-tropical Red Squirrel (Sciurus granatensis) as a pest of Cacao (Theobroma cacao L.) in the International Cacao Genebank, Trinidad; Trinidad. Tropical Agriculture (Trinidad), 85(2): 149-160.

Muñoz, A. E., y Muñoz-Santibáñez, P. (2016). Conflictos entre fauna silvestre y agricultura en Chile. Agronomía y Forestal, 53: 10-17.

Nagy-Reis, M. B., Ribeiro, M. C., Setz, E. Z. F., y Chiarello, A. G. (2019). The key role of protection status in safeguarding the ecological functions of some Neotropical mammals. Biodiversity and Conservation, 28: 25992613. doi:10.1007/s10531-019-01783-y

Navarro, J. F., y Muñoz, J. (2000). Manual de huellas de algunos mamíferos terrestres de Colombia. Medellín, Colombia: Multimpresos. $136 \mathrm{p}$.

Norris, K. (2012). Biodiversity in the context of ecosystem services: the applied need for systems approaches. Philosophical Transactions of the Royal Society B: Biological Sciences, 367: 191-199. doi:10.1098/rstb.2011.0176

Numa, C., Verdu, J. R., y Sánchez-Palomino, P. (2005). Phyllostomid bat diversity in a variegated coffee landscape. Biological Conservation, 122: 151158 .

Ortegón-Martínez, D. A., y Pérez-Torres, J. (2007). Estructura y composición del ensamblaje de murciélagos (Chiroptera) asociado a un cafetal con sombrío en la Mesa de los Santos (Santander), Colombia. Actualidades Biológicas, 29(87): 221-234. doi:10.17533/udea.acbi

Pardo-Vargas, L. E., y Payán Garrido, E. (2015). Mamíferos de un agropaisaje de palma de aceite en las sabanas inundables de Orocué, Casanare, Colombia. Biota Colombiana, 16(1): 54-66. doi:10.21068/bc.v16i1.367

Priotto, J. W. (2017). Impacto de actividades productivas sobre la diversidad, distribución y abundancia de mamíferos en Argentina. Mastozoología Neotropical, 24(2): 273-275. http://hdl.handle.net/11336/63513

Rojas Martínez, A. E., y Moreno Ortega, C. E. (2014). Los servicios ambientales que generan los mamíferos silvestres. Padi, Boletín Científico de Ciencias Básicas e Ingeniería 
del ICBI, Universidad Autónoma del Estado de Hidalgo, 2: 527-532.

Rojas-Robles, R., Stiles, F. G., y Muñoz Saba, Y. (2012). Frugivoría y dispersión de semillas de la palma Oenocarpus bataua (Arecaceae) en un bosque de los Andes colombianos. Revista de Biología Tropical, 60(4): 14451461.

Root, R. B. (1967). The niche exploitation pattern of the BlueGray Gnatcatcher. Ecological Monographs, 37: 317-350. doi: $10.2307 / 1942327$

Rosenzweig, M. L. (1981). A theory of habitat selection. Ecology, 62: 327-335.

Rumiz, D. I. (2010). Roles ecológicos de los mamíferos medianos y grandes. En: R. B, Wallace, H. Gómez, Z. R, Porcel, y D. I, Rumiz (Eds.). Distribución, ecología y conservación de los mamíferos medianos y grandes de Bolivia (pp. 53-73). Bolivia: Centro de Ecología Difusión Simón I. Patiño.

Sánchez-Clavijo, L. M., Durán, S. M., Vélez, J. G., García, R., y Botero, J. E. (2008). Estudios regionales de biodiversidad en las zonas cafeteras de Colombia. Chinchiná, Colombia: Cenicafé.

Sánchez-Clavijo, L. M., Vélez, J. G., Durán, S. M., García, R., y Botero, J. E. (2010). Estudio regional de la biodiversidad en los paisajes cafeteros de Támesis, Antioquia. Chinchiná, Colombia: Cenicafé.

Sieg, C. H. (1987). Small mammals: pests or vital components of the ecosystem. Great Plains Wildlife Damage Control Workshop Proceedings (pp. 88-92). http:// digitalcommons.unl.edu/gpwdcwp/97

Sillero-Zubiri, C., Sukumar, R., y Treves, A. (2007). Living with wildlife: the roots of conflict and the solutions. En: D. W, Mcdonald (Ed.). Key Topics in Conservation Biology (pp. 255-272). Reino Unido: Blackwell Pub. Ltd.

Solari, S., Rodríguez, J. J., Vivar, E., y Velazco, P. M. (2002). A framework for assessment and monitoring of small mammals in a lowland tropical forest. Environmental monitoring and assessment, 76: 89-104. doi:10.1023/A:1015272905263

Treves, A., y Naughton-Treves, L. (2005). Evaluating lethal control in the management of humanwildlife conflict. En: R. Woodroffe, S. Thirgood, y A. Rabinowitz (Eds.). People and Wildlife, Conflict or Coexistence? (pp. 86106). Reino Unido: Cambridge University Press.

Treves, A., Wallace, R. B., y White, S. (2009). Participatory planning of interventions to mitigate humanwildlife conflict. Conservation Biology, 23(6): 1577-1587. doi:10.1111/j.1523-1739.2009.01242.x

UNEP-WCMC, y IUCN. (2016). Protected Planet Report 2016. Cambridge (Reino Unido) y Gland (Suiza): UNEPWCMC y IUCN.

Voss, R. S. (1991). An introduction to the neotropical muroid rodent genus Zygodontomys. Bulletin of the American Museum of Natural History, 210: 1-113.

Voss, R. S., y Emmons, L. H. (1996). Mammalian diversity in Neotropical lowland rainforests: a preliminary assessment. Bulletin of the American Museum of Natural History, 230: 1-115.

Zermeño-Hernández, I., Pingarroni, A., y Martínez-Ramos, M. (2016). Agricultural land-use diversity and forest regene- ration potential in human- modified tropical landscapes. Agriculture, Ecosystems and Environment, 230: 210-220. doi:10.1016/j.agee.2016.06.007 